\title{
An audit of prostate-specific antigen and clinical symptoms in general practice
}

\author{
S Ramachandran, MC Foster, DR Thomas, AK Roalfe, RA Hall
}

\begin{abstract}
Summary
The objective was to devise local guidelines for the referral of patients with suspected prostatic carcinoma following evaluation by a retrospective audit of the value of the prostate-specific antigen concentration, together with age, urological symptoms, and digital rectal examination in the diagnosis of carcinoma of the prostate. Relevant details were collected from the notes of 582 patients from general practice and hospital. The significant diagnostic factors were ascertained by stepwise logistic regression.

Prostate-specific antigen concentration, digital rectal examination and significant terminal dribbling were the most powerful factors in the diagnosis of carcinoma of the prostate. When prostate-specific antigen concentration was considered in isolation, a value of $6.5 \mathrm{ng} / \mathrm{ml}$ appeared appropriate for referral. Age was not significant, perhaps due to the narrow patient age range. The significant diagnostic factors were built into an algorithm calculating the probability of carcinoma of the prostate. This algorithm, together with prostatespecific antigen concentration results and digital rectal examination findings, forms the basis of the referral guidelines and $a$ subsequent prospective study.
\end{abstract}

Keywords: audit; carcinoma of the prostate; prostatespecific antigen

Central Pathology Laboratory, North Staffordshire Hospital,Hartshill, Stoke on Trent, Staffordshire ST4 7PA, UK

$S$ Ramachandran

Good Hope Hospital NHS Trust, Sutton Coldfield, West Midlands, UK

MC Foster

DR Thomas

RA Hall

West Midlands

Regional Health

Authority

Birmingham, UK

AK Roalfe

Accepted 28 May 1997 an upper limit of $4 \mathrm{ng} / \mathrm{ml}$ is considered (Hybritech Tandem R Assay) in the diagnosis of prostatic carcinoma, a significant number of false negatives are seen. This can vary from $38-48 \%$ in men having clinically significant, but organ-confined neoplastic growth. ${ }^{67}$ Further, various studies show that $4-41 \%$ of men with benign prostatic hypertrophy have a PSA value above $4 \mathrm{ng} / \mathrm{ml}^{8}{ }^{8}$

Serchan and co-workers measured the PSA levels of 200 consecutive patients with benign prostatic hypertrophy and 199 consecutive men with organ-confined prostate cancer. ${ }^{9}$ The findings demonstrated that PSA was neither sensitive nor specific enough to be used as a marker of early detection of prostatic carcinoma.

In general practice, patients present with a wide variety of signs and symptoms of urological disease and often the PSA test forms the sole basis of referral of the patient to an urologist. This, together with the poor sensitivity / specificity of PSA assay, has led to a wide variety of referral patterns between different practitioners. Rectal examination in patients presenting with symptoms is another area which can contribute to this. Reports vary as to the reproducibility of rectal examination findings. Smith and Catalona, analysing the results of 116 patients who had a digital rectal examination performed twice on the same day by different urologists, concluded that the reproducibility of the findings was only fair among urologists. ${ }^{10}$ However, Varenhorst et al showed good correlation between the observation of examiners (general practitioner/ urologist) after assessment of 933 patients aged between 50 and 69 years. ${ }^{11}$

The British Prostate Group has recently published a review of prostatic disease and made various recommendations about the investigation and management of patients. ${ }^{12}$ However, this authoritative body did point out that patients with moderately elevated PSA results with little in the way of clinical symptoms, pose problems of management, which cannot be resolved by prescriptive guidelines. Instead, there would need to be local agreement between general practitioners and urologists.

We believe that the laboratory has a significant role in the formation of these guidelines. Only infrequently was our laboratory informed of the results of rectal examination or given other clinical symptoms. However, senior laboratory staff were often asked to interpret the results of the PSA test. What was the ultimate likely diagnosis to be in patients with PSA results between 4 and $40 \mathrm{ng} / \mathrm{ml}$ ? Which of the common signs and symptoms would be likely 


$$
\begin{aligned}
& \text { The diagnosis of the } 582 \text { patients after } \\
& \text { a minimum of } 18 \text { months follow-up } \\
& \text { Diagnosis } \\
& \text { - benign prostatic hypertrophy }(n=271) \\
& \text { - carcinomc of the prostate }(n=54) \\
& \text { - possible prostatitis / urinary tract infection }(n=55) \\
& \text { - non-prostatic pathology }(n=198)
\end{aligned}
$$

Box 1

to be the most helpful? Should the laboratory only report the PSA results in conjunction with clearly defined clinical information? It was to answer these questions that a comprehensive audit was undertaken with the co-operation of over 200 general practitioners and the Department of Surgery, by the Department of Biochemistry at Good Hope Hospital NHS Trust, West Midlands, UK.

\section{Methods and protocol of the audit}

The Hospital Audit Committee and the local Medical Audit Advisory Group both gave approval for the study. Hospital Ethics Committee permission was also obtained. The laboratory method of PSA analysis during the period of the audit was the Hybritech Tandem R (Hybritech, San Diego, USA) IRMA assay, which was the first commercial assay to be marketed, and is widely quoted in various other studies. The monoclonal antibody to PSA is coated onto a plastic bead. It has a linear range of $2-100 \mathrm{ng} / \mathrm{ml}$ with a quoted lower limit of detection of 0.1 to $0.2 \mathrm{ng} / \mathrm{ml}$. However, the lower limit quoted by the laboratory is $2 \mathrm{ng} / \mathrm{ml}$. All the results less than $2 \mathrm{ng} / \mathrm{ml}$ were taken as $1.9 \mathrm{ng} / \mathrm{ml}$ and similarly all results greater than $1000 \mathrm{ng} / \mathrm{ml}$ were taken as $1000.1 \mathrm{ng} / \mathrm{ml}$.

Patient details and their respective PSA results were extracted from the Laboratory 'Telepath' Database. Clinical signs, symptoms, and rectal examination findings were obtained from patient notes at general practitioner surgeries, the Family Services Health Authority, hospices and Good Hope Hospital. These details, together with investigations and diagnosis, were entered on a database using Microsoft Works for Windows, version 3. All patients who had a pre-diagnostic PSA estimation between August 1991 and December 1992 were included in the study, a period of 15 months. This resulted in a minimum of 18 months follow-up being available to establish the working diagnosis.

Of the 721 patients who had their PSA assayed during the period of the study we were able to obtain complete data in $\mathbf{5 8 2}$ patients $(80.7 \%)$. Failure in data collection was due to either the patient records not being available or the patients having moved from the locality. When multiples of the median (MoM) analyses of the PSA test results were carried out to eliminate the variability of PSA with age, the number of patients available for statistical analysis fell to 512 as the age-related median values were limited to the $40-80$ year groups.
DIAGNOSTIC CATEGORIES

The diagnosis of each of the patients after a minimum period of 18 months follow-up was taken as a fixed outcome in the statistical evaluation. The diagnosis of carcinoma of the prostate was made with histology following either biopsy or transurethral resection of the prostate except in two patients where demonstration of bony metastases via bone scan with greatly elevated PSA values over $200 \mathrm{ng} / \mathrm{ml}$ was considered adequate to make a certain clinical diagnosis. Benign prostatic hypertrophy was diagnosed with histology or continued worsening of symptoms with no indication of other prostatic pathology in the follow-up period. At the time of data collection, patients who did not fit into the above categories were categorised separately as presenting with nonprostatic pathology.

\section{Results and statistical evaluation}

DIAGNOSTIC MODELS OF OUTCOMES

In box 1, a breakdown is given of the diagnosis of the 582 patients after a minimum of 18 months follow-up. Models dependent on variables were developed by the statistician to estimate the probability of the different outcomes considered - carcinoma of the prostate and benign prostatic hypertrophy. Stepwise logistic regression analysis was carried out with variables only included if they improved the model; $\mathrm{p}<0.05$ was needed for entry. Logistic regression predicts the $\log$ transformation of the outcome probability, $\operatorname{logit}(\mathrm{p})=\log (\mathrm{p} /(1-$ p)) $=\mathrm{L}$. By substituting the characteristics of the patients into each model the probability $(p)$ of each outcome can be estimated, $p=e^{L} /\left(1+e^{L}\right)$.

Models were created by a statistician using stepwise logistic regression for each of the prostatic outcomes of interest. The algorithms of the models (equations 1-3) are found in box 2 . Equation 4 demonstrates the calculation of the probability from the previous equations; the probability of the outcome calculated by substituting $\mathrm{L}$ in equation 4 with that calculated in equations $1-3$.

Owing to the nature of the disease and the clinical and logistic implications, the diagnostic model for carcinoma of the prostate will be the only model discussed. Equation 1 indicates that the significant factors offering best fit are a positive rectal examination, suggesting carcinoma of the prostate, terminal dribbling and the PSA value. The sensitivity and the specificity for the probability levels varying between $2 \%$ and $10 \%$ are given in table 1 . Figure 1 displays the calculated probability of carcinoma of the prostate with increasing PSA levels in the presence/absence of terminal dribbling and positive rectal examination. The wide area between the lines indicates the uncertainty of an isolated PSA result in the absence of clinical information.

PSA IN ISOLATION AS A MARKER OF PROSTATIC CARCINOMA

The ability of PSA to discriminate carcinoma of the prostate was considered in isolation. Discriminatory PSA levels of 4, 5, 6, 7, 8, 9, 10 


\section{Algorithms of diagnostic models obtained using stepwise logistic regression}

Equation 4 demonstrates the conversion of equations 1-3 to probability scoring. $\mathrm{L}=$ logit (probability). In equations 1-3, 1 must be substituted for symptom / sign if it is present and 0 must be substituted if absent.

Equation 1: Carcinoma of the prostate

$\mathrm{L}=-4.3+3.27 \mathrm{X}$ (rectal exam suggesting carcinoma)

$+1.05 \mathrm{X}$ (significant terminal dribbling)

$+0.10 \times($ PSA ng/ml)

Equation 2: Benign prostatic hypertrophy

$\mathrm{L}=-4.27+2.08 \mathrm{X}$ (urological symptoms of obstruction)

$+0.91 \mathrm{X}$ (urological symptoms of irritation)

$+2.08 \mathrm{X}$ (rectal examination suggesting benign prostatic hypertrophy)

$+0.92 \times$ (rectal examination causing uncertainty)

$+0.03 \times$ (age at presentation)

$-0.02 \times($ PSA ng/ml)

Equation 3: Probable prostatitis / urinary tract infection

$\mathrm{L}=-3.4-0.75 \mathrm{X}$ (urological symptoms of obstruction)

$+1.51 \times$ (urological symptoms of irritation)

$+1.49 \times$ (presence of dysuria)

$+0.79 \times$ (rectal examination causing uncertainty)

Equation 4: $p=e^{\mathrm{L}} /\left(1+\mathrm{e}^{\mathrm{L}}\right)$

where $p=$ probability of the condition, and $L=$ logit (probability), derived in equations $1-3$. $\mathrm{ng} / \mathrm{ml}$ were selected and sensitivity and specificity calculated (see table 2). A Receiver Operated Characteristic (ROC) curve with these results demonstrates that the best compromise between sensitivity and specificity in our patient population corresponded to a PSA of $6.5 \mathrm{ng} / \mathrm{ml}$. At this level, a sensitivity of $85 \%$ and a specificity of $84 \%$ were reached. The area under the curve was 0.85 .

\section{PSA MOM IN ISOLATION AS A MARKER OF} PROSTATIC CARCINOMA

The reference range usually quoted does not consider the variability due to age. ${ }^{3}$ Median values for the age range $40-80$ years in 10 yearly increments were obtained from work carried out at the Mayo Clinic, Rochester, Minnesota, USA and the Merck Research Laboratories, Blue Bell, Pennsylvania, USA, and the multiples were calculated for each individual patient. ${ }^{3}$ The PSA MoM was considered in the same way as PSA; in isolation and also as a variable in the statistical models described previously.

PSA MoM values of between 2 and 5 were evaluated with respect to sensitivity and specificity. The ROC curve indicates that the

Table 1 Sensitivity / specificity data for probability levels of carcinoma of the prostate $(2 \%-10 \%)$ obtained by the algorithm (box 2: equation 1)

\begin{tabular}{lll}
\hline Probability (\%) & Sensitivity (\%) & Specificity (\%) \\
\hline 2 & 92.6 & 63.3 \\
4 & 87.0 & 79.0 \\
6 & 83.3 & 91.1 \\
8 & 79.6 & 93.9 \\
10 & 79.6 & 94.3 \\
\hline
\end{tabular}

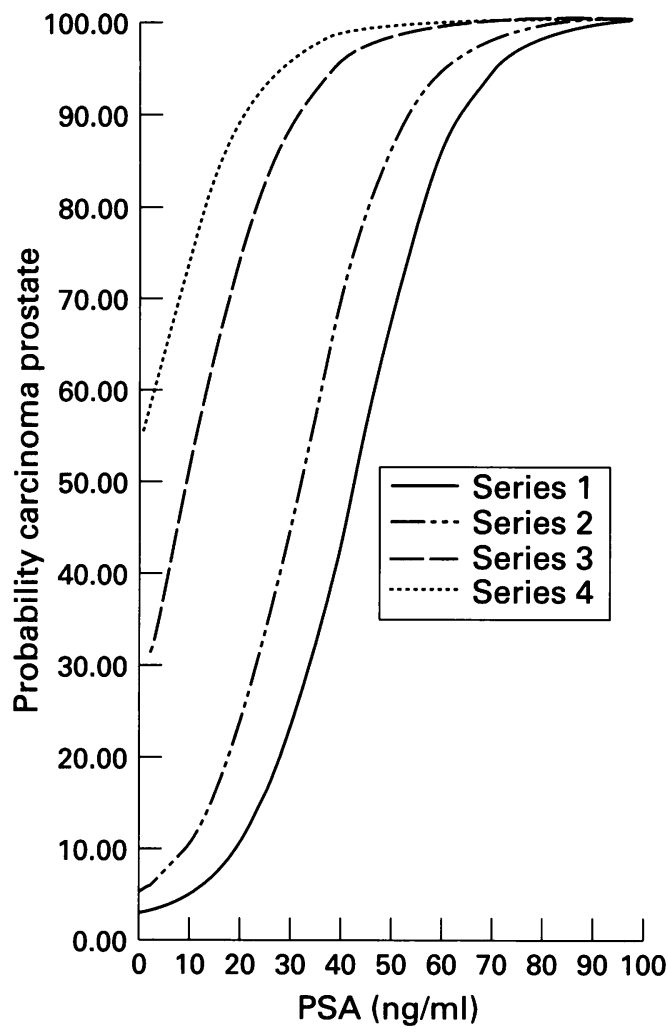

Figure 1 Relationship between the probability of carcinoma of the prostate ( $y$ axis) and PSA ( $x$ axis) in the presence / absence of the other significant findings; positive digital rectal examination and significant terminal dribbling. Series 1: rectal examination negative, terminal dribbling absent; series 2: rectal examination negative, terminal dribbling present; series 3: rectal examination positive, terminal dribbling absent; series 4: rectal examination positive, terminal dribbling present

Table 2 Sensitivity / specificity data when PSA is considered alone to diagnose carcinoma of the prostate

\begin{tabular}{lll}
\hline PSA $(\mathrm{ng} / \mathrm{ml})$ & Sensitivity (\%) & Specificity (\%) \\
\hline 4 & 88.9 & 70.0 \\
5 & 88.9 & 78.0 \\
6 & 87.0 & 82.6 \\
7 & 83.3 & 86.0 \\
8 & 83.3 & 88.3 \\
9 & 83.3 & 89.0 \\
10 & 77.8 & 90.2 \\
\hline
\end{tabular}

PSA MoM value nearest to the ideal is close to 4.5. The sensitivity at this level was $87 \%$ and the specificity was $85 \%$. The area under the curve was 0.86 . The PSA MoM appears to be marginally more discriminatory in the diagnosis of carcinoma of the prostate. However, the use of PSA MoM would limit us to the $40-80$ year age group, and a significant proportion of the patients $(12 \%)$ were over the age of 80 years. As the advantage of the PSA MoM was marginal it was not considered appropriate as a replacement for the PSA result. Further, when PSA MoM was considered with all the other variables it was not significant, thus it does not appear on the diagnostic algorithm.

\section{Discussion}

Owing to a poor predictive value of PSA and current controversies regarding treatment of 
localised prostatic carcinoma, population screening is not recommended in the UK. ${ }^{13}$ However, patients visiting their local surgeries with urological symptoms often have their PSA estimated. We undertook this audit to assess the significance of PSA in isolation and in conjunction with symptoms that may improve its discriminatory power in the diagnosis of carcinoma of the prostate.

We found PSA and PSA MoM to be similar in their predictive value. As the age-related median values were only available for the 40-80-year-old patients, and as a significant number of patients with PSA requested were over 80 years, PSA MoM estimation would result in an overall disadvantage.

The ROC curve indicates that a PSA level of $6.5 \mathrm{ng} / \mathrm{ml}$ was a suitable compromise level between sensitivity and specificity. As an action level it would lead to sensitivity of $85 \%$ and a specificity of $84 \%$ in the cases studied.

The sensitivity/specificity obtained using the algorithm (table 1) of PSA in the diagnosis of carcinoma of the prostate, could be useful in drawing up referral guidelines, and also in prioritising out-patient appointments.

A positive rectal examination had an odds ratio of 26 (95\%, confidence interval 8-83) in the diagnosis of carcinoma of the prostate. This emphasises the importance of carrying out rectal examinations, even taking into account reported variability in findings. It also demonstrates that, in the event of a positive rectal examination, referral to a urologist is usually necessary, irrespective of the PSA result. This finding was built into the referral guidelines described in figure 2.

Terminal dribbling was the other clinical finding to appear in the algorithm. This has not previously been evident. Further work is needed to confirm the significance of this finding, which has not previously been recognised as a significant symptom of prostatic carcinoma.

It was unexpected that age did not play a part in the algorithm of best fit for carcinoma of the prostate. This is perhaps explained once again by the population studied-most of the patients were in the 65 to 85 age group. This would limit the impact of age in the diagnosis of prostatic disease.

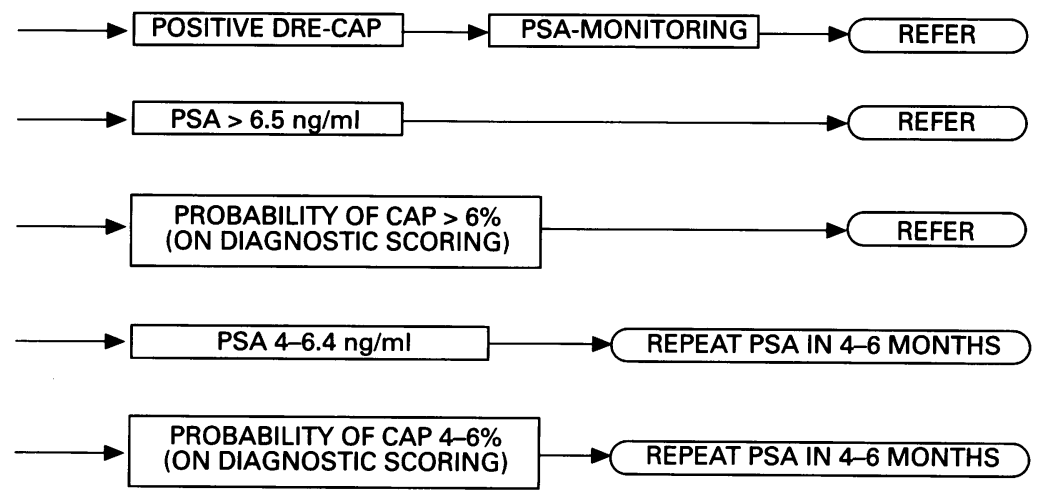

SENSITIVITY 90\% (49/54); SPECIFICITY 81\% (428/528)

Figure 2 Dynamic referral guidelines drawn up when all significant factors were considered. $\mathrm{DRE}=$ digital rectal examination; $\mathrm{CAP}=$ carcinoma of the prostate

\section{Learning points}

- PSA is elevated in carcinoma of the prostate, benign prostatic hypertrophy, acute prostatitis, acute urinary retention and prostatic

ischaemia/infarction

- laboratory methods for PSA vary. Most values quoted in the literature refer to the Hybritech methods. Local laboratories must be consulted regarding action limits

- PSA assay should only be carried out in patients with urological symptoms. Random screening yields low specificity

- rectal examination indicating carcinoma of the prostate indicates referral to a urologist irrespective of the PSA result

- PSA assay must be used in conjunction with urological symptoms (terminal dribbling was significant in our study)

- action limits must be set taking into account the local urology facilities

Box 3

An 18-month follow-up may not be adequate to diagnose small foci of neoplasm confined to the prostate. Even if the follow-up period were lengthened, this problem would not be overcome as the neoplasm may have developed subsequent to PSA measurement. This is supported by the high incidence of prostatic carcinoma. However, the aim of the audit was to establish appropriate referral guidelines for patients with symptoms needing immediate management. Clinical assessment and management should be initiated by a urologist within a few months of referral. Thus, an 18-month follow-up fulfils this criteria.

The benefit derived from treating prostatic carcinoma at present is debatable. However it is important to identify patients with prostatic carcinoma as further tests indicating specific genetic mutations are becoming available. ${ }^{14} 15$ These tests appear to identify the patients with advanced cancer and indirectly indicate the probable prognosis.

Prospective validation of the diagnostic model is essential. We would recommend using the results obtained in this study to prepare guidelines for further investigations in patients with urological symptoms. However, as in all new strategies, until validation is complete it is essential to sacrifice specificity in order to ensure maximal sensitivity. Thus, as can be seen in figure 2 , all aspects of the study have been integrated in the proposed guidelines. A level of $6 \%$ on the diagnostic model results in a sensitivity of $83.3 \%$ and a specificity of $91.1 \%$.

These guidelines have been incorporated into a database with automated suggestions depending on the risk factors described. This will also help in the periodical audit and prospective validation of the guidelines.

When the proposed guidelines were applied to the sample studied, the sensitivity was $90 \%$. The specificity, as expected, was lower at $81 \%$. Patients with a PSA level of between 4 and 6.5 $\mathrm{ng} / \mathrm{ml}$ should be assessed by their general practitioners in 4-6 months, until a full 
prospective evaluation and audit of the recommendations has been made.

Adherence to these guidelines will not change the number of patients being assessed and re-assessed by their general practitioners. However they should make referrals to the hospital urology clinic more appropriate. The guidelines can be adjusted to take account of local hospital facilities, offering great flexibility. Further audit would be automated by use

1 Lilja H. A kallikrein-like protease in prostatic fluid cleaves the predominant seminal vesicle protein. 7 Clin Invest 1985;76:1899-903.

2 Lilja H, Oldbring J, Rannevik G, Lanvell C. Seminal vesiclesecreted proteins during gelation and liquefaction of human serum. 7 Clin Invest 1987;80:281-5.

3 Oesterling JE, Cooner WH, Jacobsen SJ, Guess HA, Leiber MM. Influence of patient age on the serum PSA MM. Influence of patient age on the serum
concentrations. Urol Clin North Am 1993;20:671-80.

4 Oesterling JE. Prostate specific antigen: a critical assessment of the most useful tumor marker for adenocarcinoma of the of the most useful tumor marker

5 Glenski WJ, Malek RS, Myrtle JF. Sustained substantially increased concentration of prostate specific antigen in the absence of prostatic malignant disease: an unusual clinica scenario. Mayo Clin Proc 1992;67:249-52.

6 Hudson MA, Bahnson RR, Catalona WJ. Clinical use of prostate specific antigen in patients with prostate cancer. $\mathscr{f}$ Urol 1989;142:1011-17.

7 Lange PH, Ercole CJ, Lightner DJ. The value of serum prostate specific antigen determinations before and after radical prostatectomy. $\mathcal{F}$ Urol $1989 ; 141: 873-9$.

8 Armbruster DA. Prostate-specific antigen: biochemistry, analytical methods, and clinical application. Clin Chem 1993;39:181-95. of the database. This would be of great benefit in the local management of prostatic disease and the development of protocols as recommended by the British Prostate Group.

We wish to thank members of Medical Audit Advisory Group, all the participating General Practitioners and their Practice Staff for the cooperation provided. Thanks are also due to $\mathrm{Mr} M$ Mohamed, Department of Medical Audit, and Mr B Downing, Department of Pathology, at Good Hope Hospital for helping to set-up the database and prospective audit, respectively.

9 Sershon PD, Barry MJ, Oesterling JE. Serum PSA values in men with histologically confirmed BPH versus patients with organ-confined prostate cancer (abstract). $\mathcal{F}$ Urol 1993;149:421A.

10 Smith SS, Catalona WJ. Interexaminer variability of digita rectal examination in detecting prostatic cancer. Urology 1995;45:70-4.

11 Varenhorst E, Berglund K, Lofman O, Pedersen K Inter-observer variation in assessment of the prostate by digital rectal examination. Br 7 Urol 1993; 72:173-6.

12 Chisholm GD, Carne SJ, Fitzpatrick JM, et al. Prostate disease: management option for the primary healthcare team. Postgrad Med f 1995;71:136-42.

13 Schroder FH. Detection of prostate cancer. BMF 1995;310. 140-1.

14 Bookstein R. Tumour suppressor genes in prostatic oncogenesis. 7 Cell Biochem 1994;S19:217-23.

15 Gaddipati JP, McLeod DG, Heidenberg HB. Frequent detection of codon 877 mutation in the androgen receptor gene in advanced prostate cancer. Cancer Res 1994;54 2861-4. 